\title{
Comparison between Carotid Artery Doppler Ultrasound and Coronary Calcium Score as Predictors of Significant Coronary Artery Disease in Patients Undergoing Computed Tomography Coronary Angiography
}

Silvia Tresoldi ${ }^{\star 1}$, Riccardo Bigi ${ }^{2}$, Dario Gregori ${ }^{3}$, Anna Ravelli ${ }^{4}$, Paola Pricolo ${ }^{4}$, Nicola Flor ${ }^{1}$, Sergio Papa ${ }^{5}$ and Gianpaolo Cornalba ${ }^{6}$

${ }^{1}$ Radiologia Diagnostica ed Interventistica, Dipartimento dei Servizi Diagnostici, Azienda Ospedaliera San Paolo, Via di Rudini 8, 20142 Milano, Italy

${ }^{2}$ Cardiologia, Centro Diagnostico Italiano, Via Saint Bon 20, 20147 Milano, Italy

${ }^{3}$ Dipartimento di Medicina ambientale e Sanità Pubblica, Università di Padova, via Giustiniani 2, 35128 Padova, Italy

${ }^{4}$ Scuola di Specializzazione in Radiodiagnostica, Università degli Studi di Milano, Via Festa del Perdono 7, 20122, Milano, Italy

${ }^{5}$ Radiologia, Centro Diagnostico Italiano, Via Saint Bon 20, 20147 Milano, Italy

${ }^{6}$ Dipartimento di Scienze della Salute, Università degli Studi di Milano, Via A. di Rudinì 8, 20142 Milan, Italy

\begin{abstract}
Background: The association between carotid artery Doppler ultrasound (US) or coronary Calcium Score (CCS) and the presence of significant CAD has been suggested but not largely documented. The purpose of this study was to compare carotid artery Doppler US and CCS as predictors of significant CAD.

Methods: 56 patients ( 47 males, mean age $62 \pm 8$ years) with no history of CAD, who had undergone computed tomography coronary angiography (CTCA), Calcium Score evaluation and US, entered the study. Distribution of main socio-demographic and health related characteristics were described. On the basis of the CTCA results patients were classified as with no/non-significant CAD or with significant CAD. The presence of carotid plaques and the intima-media thickness (IMT) value were assessed with US stratifying patients into 3 groups: IMT $\leq 0.5 \mathrm{~mm}$ (free from disease); IMT 0.6-1 mm (non-significant disease); IMT >1 mm (significant disease). Volume, Mass and Agatston Score were calculated using computed tomography (CT). Considering Agatston Score absolute values patients were classified into 5 groups: Agatston Score <10; 10-99; 100-399; 400-999; $\geq 1000$; on the basis of risk percentiles patients were classified into 4 groups: $<25^{\circ}$ percentile, $<50^{\circ}$ percentile, $<75^{\circ}$ percentile, $>75^{\circ}$ percentile. Association between Calcium Score and IMT with CAD at CTCA were assessed.

Results: Age, gender, hypertension, diabetes, high blood cholesterol, familiar history of CAD and smoke habit were similar in patients with and without significant CAD, whilst chest pain was significantly $(p=0.001)$ associated with CAD. At univariate analysis, IMT $(p=0.001)$ and Calcium Score $(p<0.001)$ were associated with significant CAD. However, after adjusting for potential confounders, multivariate analysis indicated Calcium Score as the only significant and independent predictor of significant CAD.
\end{abstract}

Conclusion: Calcium Score is a more powerful marker of significant CAD compared to atherosclerotic burden of the carotid artery.

Keywords: Atherosclerosis; Coronary artery disease; Diagnostic imaging; Multi detector computed tomography; Doppler ultrasonography

Abbreviations: CAD: Coronary Artery Disease; US: Ultrasound; CCS: Coronary Calcium Score; CTCA: Computed Tomography Coronary Angiography; IMT: Intima-Media Thickness

\section{Introduction}

Cardiovascular diseases represent the first cause of death in the industrialized western world and, in the near future, it will probably be the first also all over the world [1-3]. In spite of this knowledge and notwithstanding the progress recently made in treating patients with acute cardiovascular diseases, no similar progress has been reached in secondary prevention [4]. As a matter of fact, it was seen that using only the Framingham Risk Score was effective in identifying a population at risk, but not in characterizing the individual risk [5]. If it is true that most of major cardiovascular events arise in people with many risk factors, it is also true that up to $40 \%$ (or up to $70 \%$ in young populations) of patients with myocardial infarction would have not been labelled as "patients at risk" by utilizing the present predicting models (Framingham Score or Procam Score), and sudden death, as the primary manifestation of cardiac pathology, hits $20 \%$ of patients [1].

The early identification of asymptomatic coronary artery disease
(CAD) represents a basic target in the field of secondary prevention. In particular, the selection of a non-invasive diagnostic technique that could be used as a screening tool, sufficiently accurate and costeffective, represents a major target in cardiology. Several studies have underlined the association between carotid artery Doppler ultrasound (US) or coronary Calcium Score (CCS) and the presence of significant CAD [5-8]. However, few data on direct comparison between the two methods exist in literature $[5,9,10]$.

The aim of our study was to compare the capability of carotid artery

*Corresponding author: Silvia Tresoldi, Dipartimento di Radiologia Diagnostica ed Interventistica, Azienda Ospedaliera San Paolo, Italy, Tel: +39 3333111021 Fax: +39 02 50323393; E-mail: silvia.3soldi@gmail.com

Received February 23, 2014; Accepted March 15, 2014; Published March 24 2014

Citation: Tresoldi S, Bigi R, Gregori D, Ravelli A, Pricolo P, et al. (2014) Comparison between Carotid Artery Doppler Ultrasound and Coronary Calcium Score as Predictors of Significant Coronary Artery Disease in Patients Undergoing Computed Tomography Coronary Angiography. Cardiol Pharmacol 3: 116. doi:10.4172/23296607.1000116

Copyright: ( 2014 Tresoldi S, et al. This is an open-access article distributed under the terms of the Creative Commons Attribution License, which permits unrestricted use, distribution, and reproduction in any medium, provided the original author and source are credited. 
Citation: Tresoldi S, Bigi R, Gregori D, Ravelli A, Pricolo P, et al. (2014) Comparison between Carotid Artery Doppler Ultrasound and Coronary Calcium Score as Predictors of Significant Coronary Artery Disease in Patients Undergoing Computed Tomography Coronary Angiography. Cardiol Pharmacol 3: 116. doi:10.4172/2329-6607.1000116

Doppler US and CCS to predict the presence of significant CAD in patients without known cardiovascular disease.

\section{Materials and Methods}

\section{Patient Population}

The sample used come from the patients referred to our Centre to undergo computed tomography coronary angiography (CTCA) in the period between January 2009 and May $2010(n=136)$ retrospectively selected. We excluded patients with history of CAD (myocardial infarction, previous percutaneous or surgical revascularization). Among the remaining patients we selected the ones who underwent also carotid artery Doppler US with a maximum interval of 1 month between the two examinations. Finally, the study population was composed of 56 patients (47 males and 9 females; mean age \pm SD: 62 \pm 8 years; median age: 62 years; age range $41-88$ years); their clinical features, collected in a specific form at the time of the exam, are summarized in Table 1 . Table 2 shows clinical indications to CTCA for patients selected.

\section{CT coronary angiography}

CT coronary angiography examinations were performed using a Dual Source CT scan (Somaton Definition, Siemens, Forcheim, Germany), with retrospective ECG gating (automatic dose modulation), during intravenous administration of iodine contrast media (Iopamidol $370 \mathrm{mgI} / \mathrm{ml}, 75-85 \mathrm{ml}$ ) at $5 \mathrm{ml} / \mathrm{sec}$ followed by the administration of a mixed bolus of contrast media (20\%) and saline solution $(80 \%)$ at the same injection rate, using the bolus test $(10 \mathrm{ml}$

\begin{tabular}{|c|c|c|}
\hline Feature & patients $\mathbf{( n}^{\mathbf{}} \mathbf{)}$ & patients (\%) \\
\hline Patients & 56 & 100 \\
\hline Males & 47 & 84 \\
\hline Females & 9 & 16 \\
\hline Age (years) & $62 \pm 8$ & \\
\hline Risk factors & & \\
\hline Hypertension $^{*}$ & 33 & 59 \\
\hline Hypercholesterolemia & 30 & 54 \\
\hline Smoke & 21 & 38 \\
\hline Family history & 18 & 32 \\
\hline Diabetes $^{\text {Obesity }}$ & 6 & 11 \\
\hline Symptoms $^{\circ}$ & 0 & 0 \\
\hline
\end{tabular}

*Risk factors were defined according to the "Framingham Risk Score" [2] ${ }^{\circ}$ Atypical pain, dyspnea, precordial pain

Table 1: Clinical features of the study population.

\begin{tabular}{|l|c|c|}
\hline Indication & $\begin{array}{c}\text { Patients } \\
\left(\mathbf{n}^{\circ}\right)\end{array}$ & $\begin{array}{c}\text { patients } \\
(\mathbf{\%})\end{array}$ \\
\hline $\begin{array}{l}\text { Doubtful or inconclusive provocative test in asymptom- } \\
\text { atic patient }\end{array}$ & 31 & 55.4 \\
\hline $\begin{array}{l}\text { Doubtful or inconclusive provocative test in patient with } \\
\text { atypical pain }\end{array}$ & 1 & 1.8 \\
\hline Positive provocative test in patient with atypical pain & 2 & 3.6 \\
\hline $\begin{array}{l}\text { Discrepancy between the results of two different pro- } \\
\text { vocative tests }\end{array}$ & 5 & 8.9 \\
\hline Multiple cardiovascular risk factors & 4 & 7.1 \\
\hline Episodes of atypical pain & 8 & 14.3 \\
\hline Sleep apneae & 1 & 1.8 \\
\hline Known atherosclerotic disease in other districts & 4 & 7.1 \\
\hline Total & 56 & 100 \\
\hline
\end{tabular}

Table 2: Clinical indications to CT coronary angiography in the 56 patients selected. of contrast media) technique. The acquisition parameters were: tube voltage $100-120 \mathrm{kVp}$, tube current $700 \mathrm{mAs}$, cranio-caudal acquisition from the carena to the diaphragm, automatic pitch variation.

Oral beta-blockers (50-100 mg metoprolol) one hour before the test, if no contraindicated, were given to the patients with cardiac rhythm $>65$ beats per minute (bpm).

In case of persistence of high but stable cardiac rhythm (65-70 bpm after beta-blockers administration), the examination was performed as well. Moreover, sublingual nitrates (nitroglycerine $0.30 \mathrm{mg}$ ) were administered to all the patients just before the examination.

All the examinations have been interpreted by a radiologist certified in non-invasive cardiac imaging, in blind respect to the Doppler US evaluation of carotid IMT. On the basis of the CTCA results all the patients were classified into two different groups: patients with no or non-significant coronary artery disease (stenosis $<50 \%)$ and patients with significant coronary artery disease (at least one stenosis $\geq 50 \%$ ) [11].

\section{Carotid artery Doppler US}

Carotid artery examination was performed with a GE Logiq 9 ultrasound system (GE Healthcare, Little Chalfont, Buckinghamshire, $\mathrm{UK}$ ), with an $8 \mathrm{MHz}$ linear probe. The protocol used to obtain images was consistent with the American Society of Echocardiography recommendations [12] and with the guidelines of the Società Italiana di Diagnostica Vascolare (SIDV) [13].

On longitudinal images obtained using B-mode ultrasonography, the maximum and the overall mean IMT value of the common carotid artery and the presence of carotid plaques (defined as isolated and focal areas of abnormal intima protruding into the lumen more than $1.5 \mathrm{~mm}$ or at least $50 \%$ of the surrounding IMT value) [12], were evaluated. IMT represents the thickness of the intima plus the media component of the vessel wall; three measurements were performed on the far wall of both common carotid arteries, $1 \mathrm{~cm}$ below the carotid bulb, along a straight arterial segment of $10 \mathrm{~mm}$ length (Figures 1 and 2). Patients were stratified into 3 groups according to the IMT value: patients with

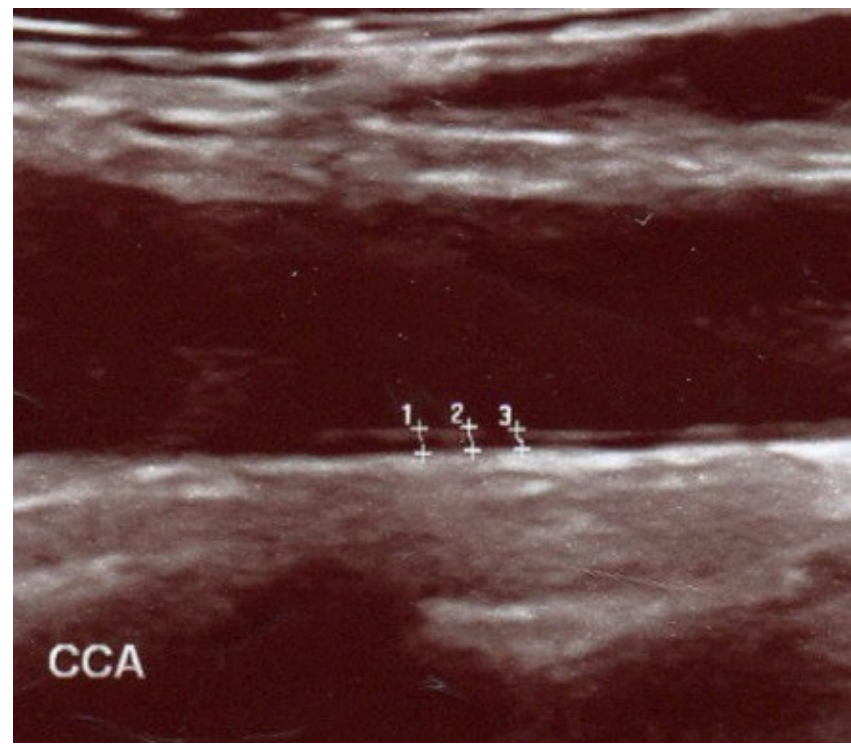

Figure 1: 41 years-old IMT evaluation. B-mode US longitudinal image showing normal IMT value $(0.5 \mathrm{~mm})$ measured on the far wall of the right common carotid artery (CCA) about $1 \mathrm{~cm}$ below the carotid bulb, along a straight arterial segment of $10 \mathrm{~mm}$ length. 
Citation: Tresoldi S, Bigi R, Gregori D, Ravelli A, Pricolo P, et al. (2014) Comparison between Carotid Artery Doppler Ultrasound and Coronary Calcium Score as Predictors of Significant Coronary Artery Disease in Patients Undergoing Computed Tomography Coronary Angiography. Cardiol Pharmacol 3: 116. doi:10.4172/2329-6607.1000116

IMT $<0.5 \mathrm{~mm}$ were considered free from disease; patients with IMT between 0.6-1 mm were considered affected by non-significant disease; patients with IMT $>1 \mathrm{~mm}$ were considered affected by significant disease $[14,15]$. Therefore, we considered carotid atherosclerosis either the presence of plaques or a IMT $>1 \mathrm{~mm}[4,16]$.

\section{Calcium score}

Coronary Calcium Score data were acquired through 64-slice dual source CT scan (Somatom Definition, Siemens, Forcheim, Germany) with the following acquisition parameters: tube voltage $120 \mathrm{kVp}$, tube current $80 \mathrm{mAs}$, prospective ECG triggering and acquisition window at $70 \%$ of the R-R interval. No contrast media administration was

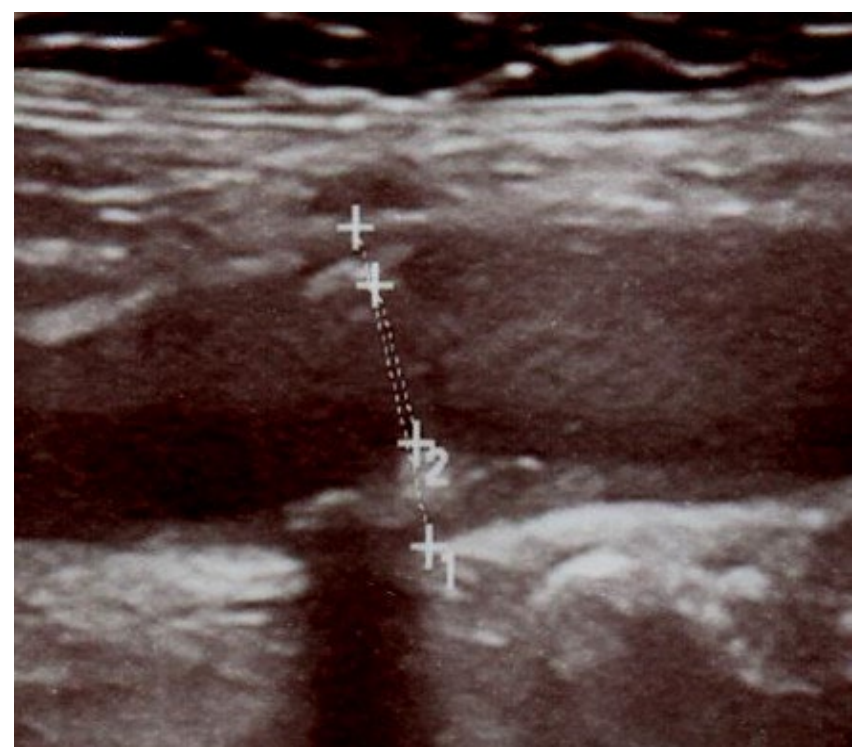

Figure 2: 74 years-old IMT evaluation. B-mode US longitudinal image showing a calcific plaque at the right carotid bulb. required and the acquisition was performed with patient holding his breath. Volume, Mass and Agatston Score were calculated using the dedicated software (Figure 3). Considering Agatston Score absolute values patients were classified into 5 groups: Agatston Score $<10$; 10 99; 100-399; 400-999; $\geq 1000$; on the basis of risk percentiles patients were classified into 4 groups: $<25^{\circ}$ percentile, $<50^{\circ}$ percentile, $<75^{\circ}$ percentile, $>75^{\circ}$ percentile. Patients with Agatston Score $>1000$ did not undergo CTCA following the Centre guidelines and were not included in the study; in all the other individuals of our sample CTCA was then performed.

\section{Statistical analysis}

Distribution of main socio-demographic and health related characteristics were described. Continuous variables were expressed as mean and standard deviation and categorical variables as percentages and absolute numbers. Differences between groups were compared using Wilcoxon and Pearson Chi-Square test or Fisher's exact test, as appropriate. Association of "CT coronary angiography" and "carotid artery Doppler US” variables was investigated using a multivariable logistic regression model, adjusted by relevant covariates. All variables considered were entered into the model "as is", i.e. without any transformation or cut-off. If significant non-linearity using a score test was present, the specific covariate's effect was modeled using a restricted cubic spline. Selection criteria was the AIC (Akaike Information Criterion) applied backward for selecting significant covariates. First, the base model was selected using only covariates' information. Then, "CT coronary angiography" and "carotid artery Doppler US" variables were considered producing the final model, selected if superior in terms of AIC at a significance level of 0.05 . P-values have been explicitly indicated if below the 0.25 threshold, otherwise the "NS" indication was used. To account for possible over fitting in the regression model secondary to high ratio between covariates and events, cross-validation and bootstrap (200 runs) techniques were applied. For the logistic regression model, Somer's concordance Index Dxy (the closer to one in absolute value the better) were obtained and evaluated for this purpose. Multivariable Odds Ratios are presented along with their $95 \%$
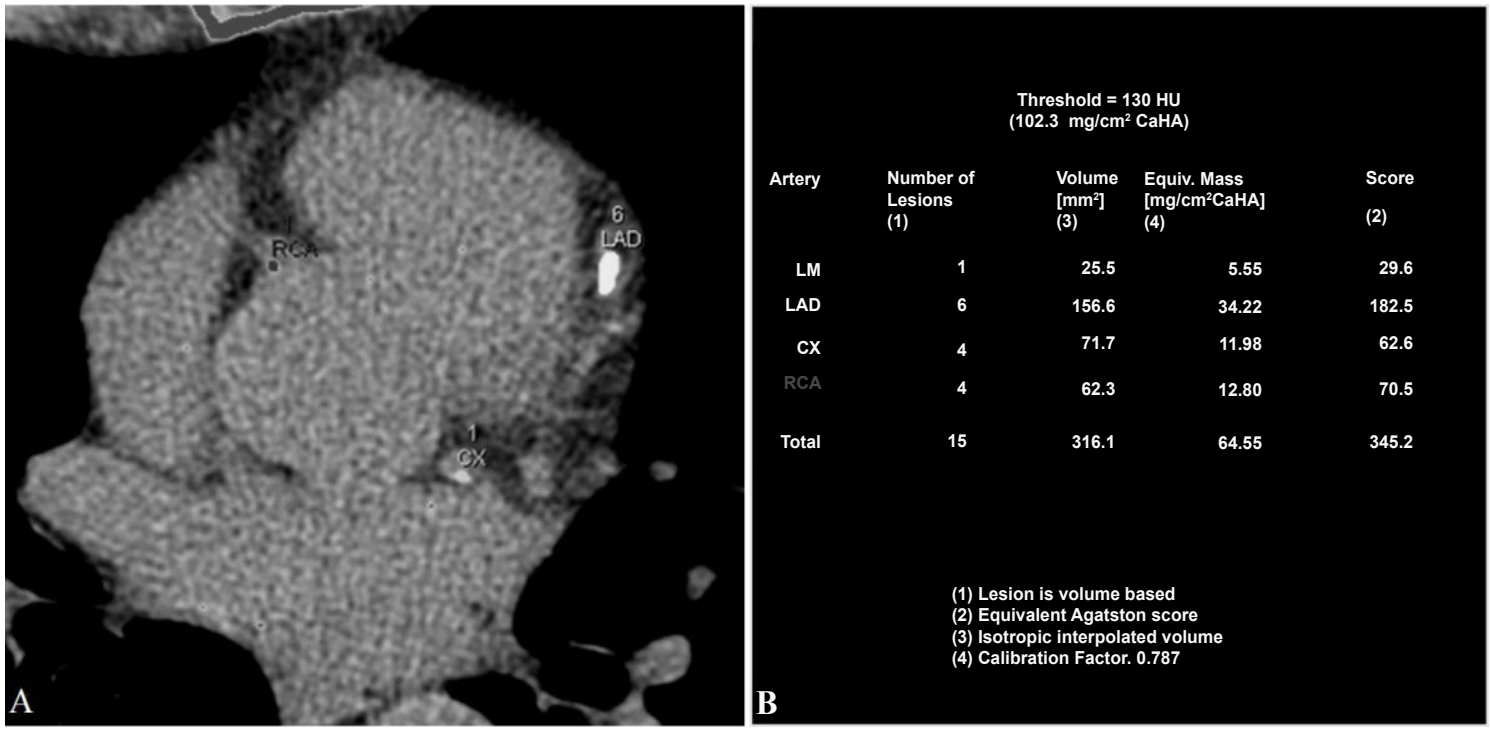

Figure 3: Calcium Score evaluation in a 71 years old male. a) ECG-gated axial CT image at a level corresponding to the origin of the right coronary artery (RCA), showing calcified plaques automatically detected by the software, in RCA, left anterior descending artery (LAD) and circumflex artery (CX). b) Calcium Score report with details about number of lesions, Volume, Mass and Agatston Score for each artery under study and in total. 
Citation: Tresoldi S, Bigi R, Gregori D, Ravelli A, Pricolo P, et al. (2014) Comparison between Carotid Artery Doppler Ultrasound and Coronary Calcium Score as Predictors of Significant Coronary Artery Disease in Patients Undergoing Computed Tomography Coronary Angiography. Cardiol Pharmacol 3: 116. doi:10.4172/2329-6607.1000116

Page 4 of 6

confidence intervals. The statistical significance was settled at $\mathrm{p}<0.05$. The R System (release 2.7.0) statistical package and the Harrell's Design and Hmisc libraries were used for analysis.

\section{Results}

\section{CT coronary angiography}

Twenty-three percent of the patients $(13 / 56)$ had significant coronary artery disease, $54 \%$ (30/56) had non-significant stenosis in one or more epicardial vessels and $23 \%(13 / 56)$ had patent coronary arteries.

\section{IMT and Calcium Score}

No patients had significant carotid artery stenosis, $88 \%(49 / 56)$ of the patients had non-significant disease, $12 \%(7 / 56)$ had no carotid disease. Considering absolute Calcium Score values 21\% (12/56) of the patients had Agatston Score 0, 14\% (8/56) had Agatston Score <10; $21 \%(12 / 56)$ between $10-99 ; 23 \%$ (13/56) between $100-399$, while $20 \%$ $(11 / 56)$ had Agatston Score $>400$.

Agatston Score absolute values $>400$ are associated with high likelihood of significant coronary artery disease $[1,17]$.

Table 3 summarizes IMT and Calcium Score percentiles distributions in the studied population.

\section{Significant CAD predictors}

At univariate analysis IMT and Calcium Score resulted significantly associated with significant CAD. However, after adjusting for potential confounders, multivariate analysis indicated Calcium Score as the only significant and independent predictor of significant CAD. Table 4 summarizes the results of the univariate analysis for prediction of significant coronary artery disease. Among clinical variables only chest pain resulted significantly related to the presence of CAD. On the contrary, all the instrumental variables, either Doppler-US or CT based, representing the global atherosclerotic burden, resulted significant predictors of CAD. However, after adjusting for potential confounders using a multivariate analysis, only the Calcium Score remained a significant and independent predictor (OR 1.55, 95\%; unit increase C.I.1.04-2.31).

\section{Discussion}

The relationship between peripheral and coronary atherosclerosis is well documented and, actually, various imaging techniques are capable of identifying the atherosclerosis of the carotid district; these methods have been employed as indirect index of coronary disease $[6,18,19]$. As a matter of fact, it has been demonstrated that the carotid IMT can be

\begin{tabular}{|l|c|c|}
\hline $\mathbf{I M T}(\mathbf{m m})$ & patients $\left.\mathbf{( n}^{\circ}\right)$ & patients $\mathbf{( \% )}$ \\
\hline$<0.5$ & 1 & 2 \\
\hline $0.6-1$ & 22 & 39 \\
\hline$>1$ & 33 & 59 \\
\hline & 56 & 100 \\
\hline Calcium Score & & \\
\hline$<25^{\circ}$ & 20 & 36 \\
\hline$<50^{\circ}$ & 8 & 14 \\
\hline$<75^{\circ}$ & 16 & 29 \\
\hline$>75^{\circ}$ & 12 & 21 \\
\hline & 56 & 100 \\
\hline
\end{tabular}

Table 3: Intima-media-thickness and Calcium Score distributions in the studied population.

\begin{tabular}{|c|c|c|c|}
\hline & $\begin{array}{c}\text { No CAD } \\
(\mathrm{n}=43)\end{array}$ & $\begin{array}{c}\text { CAD } \\
(\mathrm{n}=13)\end{array}$ & $\mathrm{p}$ \\
\hline Age & $62(55-69)$ & $63(61-65)$ & $0.56^{*}$ \\
\hline Male gender & $37(86 \%)$ & $10(77 \%)$ & $0.43^{\circ}$ \\
\hline Smoke & $37(16 \%)$ & $5(38 \%)$ & $0.93^{\circ}$ \\
\hline Family history & $14(33 \%)$ & $4(31 \%)$ & $0.90^{\circ}$ \\
\hline Diabetes & $5(12 \%)$ & $1(8 \%)$ & $0.68^{\circ}$ \\
\hline Hypertension & $23(53 \%)$ & $10(77 \%)$ & $0.13^{\circ}$ \\
\hline Chest pain & $7(16 \%)$ & $8(62 \%)$ & $\mathbf{0 . 0 0 1}^{\circ}$ \\
\hline Hypercholesterolemia & $21(49 \%)$ & $9(69 \%)$ & $0.19^{\circ}$ \\
\hline Significant carotid artery stenosis & $36(84 \%)$ & $13(100 \%)$ & $0.12^{\circ}$ \\
\hline Maximum IMT & $1(0.8-1.2)$ & $1.3(1.2-1.4)$ & $\mathbf{0 . 0 0 1}^{*}$ \\
\hline Common carotid artery IMT & $1(0.8-1.2)$ & $1.2(1.1-1.3)$ & $\mathbf{0 . 0 1 2}^{*}$ \\
\hline Agatston Score & $11(0-60)$ & $411(227-506)$ & $<\mathbf{0 . 0 0 1}^{*}$ \\
\hline Plaques volume & $6-85(0-41)$ & $345(176-382)$ & $<\mathbf{0 . 0 0 1}^{*}$ \\
\hline Plaques mass & $2.1(0-10)$ & $72(45-92)$ & $<\mathbf{0 . 0 0 1}^{*}$ \\
\hline
\end{tabular}

$\mathrm{CAD}=$ coronary artery disease

*Fisher's exact test

${ }^{\circ}$ Pearson Chi-square test

Table 4: Univariate predictors of significant coronary artery disease.

considered an additional risk factor not only for ischemic stroke but also for myocardial infarction $[4,6,18,20]$.

Thus, carotid artery Doppler US and coronary Calcium Score are able to identify the atherosclerotic disease at pre-clinical stage, but even if both such methods can identify the sub-clinical disease, the correlation between them is weak, probably because the coronary calcifications represent a more advanced stage of vascular disease [5].

The cardiovascular screening strategy suggested by Naghavi et al. in the SHAPE guidelines proposes a stratification of the patients initially based on the evaluation of Calcium Score or of the carotid IMT values [3]. As a matter of fact there are, by now, many evidences that these two non-invasive imaging techniques are able to furnish additional information to the traditional methods of risk stratification [2,14,20-25]. Our data, in accordance with literature, suggest that the markers of atherosclerotic burden, both carotid (IMT) and coronary (CS), are more accurate than risk factors only or obstructive carotid disease in identifying subjects at risk [1]. On one hand, the results of the EDUCATE (Early Detection by Ultrasound of Carotid Artery Intima-media Thickness Evaluation) study demonstrate that there is an association between carotid atherosclerosis, significant coronary artery disease and incidence of major and minor cardiovascular events and that the evaluation of the carotid atherosclerosis can have an additional value to the risk evaluation $[4,25]$. On the other hand, already from studies with electron beam CT, even Calcium Score proved to be able to predict, independently and in a more accurate way than the risk factors only, the major cardiovascular events in populations of patients at low, medium and high risk of events [7,22,24,26].

Some authors however, and particularly Folsom, underline how Calcium Score resulted to be the best predictor for coronary heart disease and total cardiovascular disease, while IMT resulted to be slightly better than Calcium Score only in predicting cerebrovascular events [9]. These authors suggest, particularly in patients at medium risk (Framingham), to prefer Calcium Score to IMT in evaluating coronary risk [9]. In accordance with said data, in our sample, after correcting for confounding variables (risk profile), the local marker (Calcium Score) remains the only independent and significant predictor of significant CAD, unlike the remote marker (IMT) that does not result as independent predictor at the multivariate analysis. This could likely 
Citation: Tresoldi S, Bigi R, Gregori D, Ravelli A, Pricolo P, et al. (2014) Comparison between Carotid Artery Doppler Ultrasound and Coronary Calcium Score as Predictors of Significant Coronary Artery Disease in Patients Undergoing Computed Tomography Coronary Angiography. Cardiol Pharmacol 3: 116. doi:10.4172/2329-6607.1000116

Page 5 of 6

be attributed to the fact that the IMT becomes more predictive at more advanced age $[9,10]$.

Evidences in literature are based on perspective studies on numerous populations, where risk evaluation is obtained by monitoring events during the follow-up or on studies which evaluate the presence of CAD at coronary angiography examination $[8,9,25]$. The evaluation of the presence of disease at coronary angiography examination, considering its invasiveness, makes it that the examined patients, sent to coronary angiography, even if only with a diagnostic aim, may result less representative of the general population at risk of CAD. The advent, in the last decade, of the CTCA with the possibility to study coronaries by means of non-invasive imaging, of which the accuracy and the high negative predictive value are more and more confirmed [27-29], can represent a turning-point in the study of subjects at low to medium risk of coronary artery disease. Our data therefore, being based on a population of patients sent to CTCA, and not to conventional coronary angiography, in our opinion, can better represent the actual target population of the secondary prevention.

This study has some limits the main of which is certainly represented by the scarce numerousness of the sample. Moreover, we have to point out that at our Centre we do not perform CTCon patients with Calcium Score $>1000$ and therefore some patients potentially at high risk might have not been included in the study population. A further limit of the study may be due to the time of execution of the examinations: in fact the evaluation of Calcium Score was made at the same time of the CTCA, while the carotid artery Doppler US could have been made even 1 month before or later, and this might have influenced the possible greater accuracy of CCS compared to the IMT evaluation.

In conclusion, in accordance with our results, in a population at medium risk of $\mathrm{CAD}$, the markers of carotid atherosclerosis burden are less significant and only partially useful in predicting the presence of obstructive coronary pathology and cannot represent a substitute of the markers of local atherosclerotic burden.

\section{References}

1. Becker A, Leber AW, Becker C, von Ziegler F, Tittus J, et al. (2008) Predictive value of coronary calcifications for future cardiac events in asymptomatic patients with diabetes mellitus: a prospective study in 716 patients over 8 years. BMC Cardiovasc Disord 8: 27.

2. Wilson PW, D’Agostino RB, Levy D, Belanger AM, Silbershatz H, et al. (1998) Prediction of coronary heart disease using risk factor categories. Circulation 97: 1837-1847

3. Naghavi M, Falk E, Hecht HS, Jamieson MJ, Kaul S, et al. (2006) From vulnerable plaque to vulnerable patient--Part III: Executive summary of the Screening for Heart Attack Prevention and Education (SHAPE) Task Force report. Am J Cardiol 98: $2 \mathrm{H}-15 \mathrm{H}$.

4. Akosah KO, McHugh VL, Barnhart SI, Schaper AM, Mathiason MA et al (2006) Carotid ultrasoud for risk clarification in young to middle-aged adults undergoing elective coronary angiography. Am J Hypertens 19:1256-1261.

5. Lester SJ, Eleid MF, Khandheria BK, Hurst RT (2009) Carotid intima-media thickness and coronary artery calcium score as indications of subclinical atherosclerosis. Mayo Clin Proc 84: 229-233.

6. Belhassen L, Carville C, Pelle G, Monin JL, Teiger E, et al. (2002) Evaluation of carotid artery and aortic intima-media thickness measurements for exclusion of significant coronary atherosclerosis in patients scheduled for heart valve surgery. J Am Coll Cardiol 39: 1139-1144.

7. Arad Y1, Goodman KJ, Roth M, Newstein D, Guerci AD (2005) Coronary calcification, coronary disease risk factors, C-reactive protein, and atherosclerotic cardiovascular disease events: the St. Francis Heart Study. J Am Coll Cardiol 46: 158-165.

8. Bampi AB, Rochitte CE, Favarato D, Lemos PA, da Luz PL (2009) Comparison of non-invasive methods for the detection of coronary atherosclerosis. Clinics (Sao Paulo) 64: 675-682.

9. Folsom AR, Kronmal RA, Detrano RC, O'Leary DH, Bild DE, et al. (2008) Coronary artery calcification compared with carotid intima-media thickness in the prediction of cardiovascular disease incidence: the Multi-Ethnic Study of Atherosclerosis (MESA). Arch Intern Med 168: 1333-1339.

10. Newman AB, Naydeck BL, Ives DG, Boudreau RM, Sutton-Tyrrell K, et al. (2008) Coronary artery calcium, carotid artery wall thickness, and cardiovascular disease outcomes in adults 70 to 99 years old. Am J Cardiol 101: 186-192.

11. Mowatt G, Cook JA, Hillis GS, Walker S, Fraser C, et al. (2008) 64-Slice computed tomography angiography in the diagnosis and assessment of coronary artery disease: systematic review and meta-analysis. Heart 94: 13861393.

12. Stein JH, Korcarz CE, Hurst RT, Lonn E, Kendall CB, et al. (2008) Use of carotid ultrasound to identify subclinical vascular disease and evaluate cardiovascular disease risk: a consensus statement from the American Society of Echocardiography Carotid Intima-Media Thickness Task Force. Endorsed by the Society for Vascular Medicine. J Am Soc Echocardiogr 21: 93-111.

13. Antignani PL, Benedetti-Valentini F, Aluigi L, Baroncelli TA, Camporese G, et al (2012) Diagnosis of vascular diseases. Ultrasound investigations--guidelines. Int Angiol 31: 1-77.

14. Bots ML, Hoes AW, Koudstaal PJ, Hofman A, Grobbee DE (1997) Common carotid intima-media thickness and risk of stroke and myocardial infarction: the Rotterdam Study. Circulation 96: 1432-1437.

15. MITCHELL JR, SCHWARTZ CJ (1962) Relationship between arterial disease in different sites. A study of the aorta and coronary, carotid, and iliac arteries. Br Med J: 1293-1301.

16. Albers GW, Hart RG, Lutsep HL, Newell DW, Sacco RL (1999) AHA Scientific Statement. Supplement to the guidelines for the management of transient ischemic attacks: A statement from the Ad Hoc Committee on Guidelines for the Management of Transient Ischemic Attacks, Stroke Council, American Heart Association. Stroke 30: 2502-2511.

17. Oudkerk M, Stillman AE, Halliburton SS, Kalender WA, Möhlenkamp S, et al. (2008) Coronary artery calcium screening: current status and recommendations from the European Society of Cardiac Radiology and North American Society for Cardiovascular Imaging. Eur Radiol 18: 2785-2807.

18. de Groot E, Hovingh GK, Wiegman A, Duriez P, Smit AJ, et al. (2004) Measurement of arterial wall thickness as a surrogate marker for atherosclerosis. Circulation 109: III33-38.

19. Ibañez B, Badimon JJ, Garcia MJ (2009) Diagnosis of atherosclerosis by imaging. Am J Med 122: S15-25.

20. O'Leary DH, Polak JF, Kronmal RA, Manolio TA, Burke GL, et al. (1999) Carotidartery intima and media thickness as a risk factor for myocardial infarction and stroke in older adults. Cardiovascular Health Study Collaborative Research Group. N Engl J Med 340: 14-22.

21. Persson J, Formgren J, Israelsson B, Berglund G (1994) Ultrasounddetermined intima-media thickness and atherosclerosis. Direct and indirect validation. Arterioscler Thromb 14: 261-264.

22. Shaw LJ, Raggi P, Schisterman E, Berman DS, Callister TQ (2003) Prognostic value of cardiac risk factors and coronary artery calcium screening for all-cause mortality. Radiology 228: 826-833.

23. Greenland P, LaBree L, Azen SP, Doherty TM, Detrano RC (2004) Coronary artery calcium score combined with Framingham score for risk prediction in asymptomatic individuals. JAMA 291: 210-215

24. Taylor AJ, Bindeman J, Feuerstein I, Cao F, Brazaitis M, et al. (2005) Coronary calcium independently predicts incident premature coronary heart disease over measured cardiovascular risk factors: mean three-year outcomes in the Prospective Army Coronary Calcium (PACC) project. J Am Coll Cardiol 46: 807-814.

25. Akosah KO, McHugh VL, Barnhart SI, Mathiason MA, Schaper AM, et al (2007) Pilot Results of the Early Detection by Ultrasound of Carotid Artery Intima-Media Thickness Evaluation (EDUCATE) study. Am J Hypertens 20: 1183-1188.

26. Budoff MJ, Diamond GA, Raggi P, Arad Y, Guerci AD et al. (2002) Continuous probabilistic prediction of angiographically significant coronary artery disease using electron beam tomography. Circulation 105: 1791-1796. 
Citation: Tresoldi S, Bigi R, Gregori D, Ravelli A, Pricolo P, et al. (2014) Comparison between Carotid Artery Doppler Ultrasound and Coronary Calcium Score as Predictors of Significant Coronary Artery Disease in Patients Undergoing Computed Tomography Coronary Angiography. Cardiol Pharmacol 3: 116. doi:10.4172/2329-6607.1000116

Page 6 of 6

27. Paech DC, Weston AR (2011) A systematic review of the clinical effectiveness of 64-slice or higher computed tomography angiography as an alternative to invasive coronary angiography in the investigation of suspected coronary artery disease. BMC Cardiovasc Disord 11: 32

28. Gueret P, Deux JF, Bonello L, Sarran A, Tron C, et al. (2013) Diagnostic performance of computed tomography coronary angiography (from the
Prospective National Multicenter Multivendor EVASCAN Study). Am J Cardiol 111: 471-478.

29. Sajjadieh A, Hekmatnia A, Keivani M, Asoodeh A, Pourmoghaddas M, et al. (2013) Diagnostic performance of 64-row coronary CT angiography in detecting significant stenosis as compared with conventional invasive coronary angiography. ARYA Atheroscler 9: 157-163. 